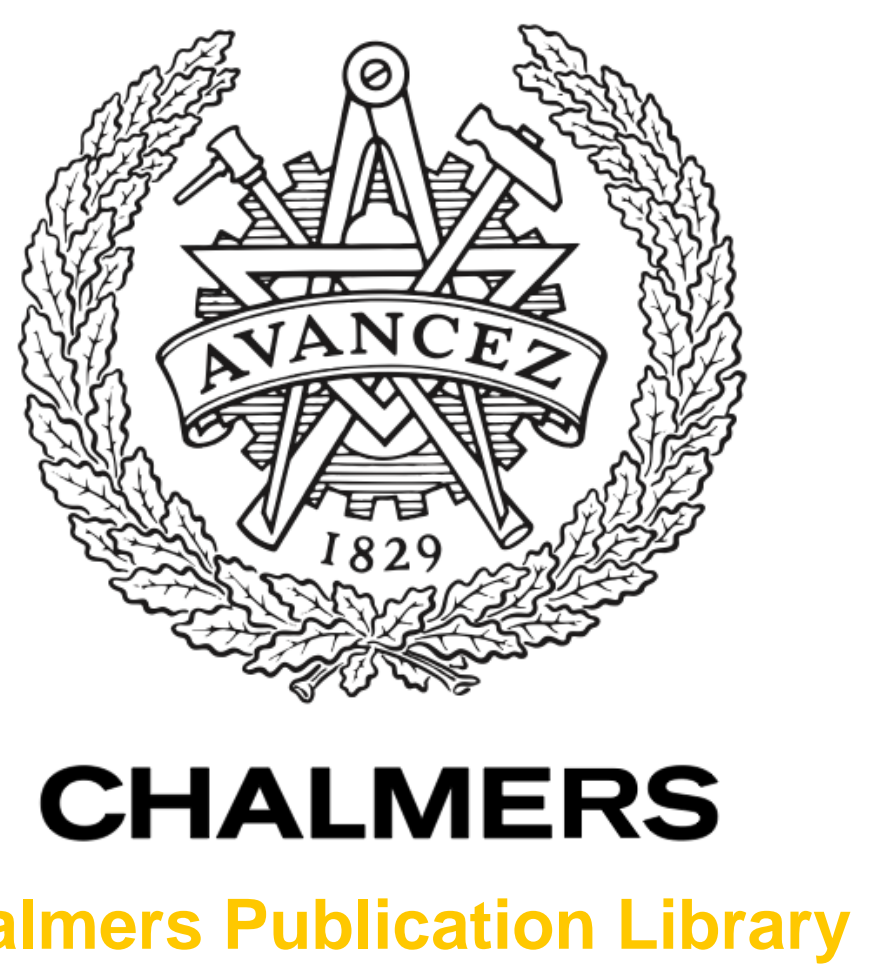

Numerical Sensitivity of Linear Matrix Inequalities Using Shift and Delta Operators

This document has been downloaded from Chalmers Publication Library (CPL). It is the author's version of a work that was accepted for publication in:

IEEE Transactions on Automatic Control (ISSN: 0018-9286)

Citation for the published paper:

Lennartson, B. ; Middleton, R. ; Gustafsson, I. (2012) "Numerical Sensitivity of Linear Matrix Inequalities Using Shift and Delta Operators". IEEE Transactions on Automatic

Control, vol. 57(11), pp. 2873-2879.

http://dx.doi.org/10.1109/TAC.2012.2192361

Downloaded from: http://publications.lib.chalmers.se/publication/171156

Notice: Changes introduced as a result of publishing processes such as copy-editing and formatting may not be reflected in this document. For a definitive version of this work, please refer to the published source. Please note that access to the published version might require a subscription.

Chalmers Publication Library (CPL) offers the possibility of retrieving research publications produced at Chalmers University of Technology. It covers all types of publications: articles, dissertations, licentiate theses, masters theses, conference papers, reports etc. Since 2006 it is the official tool for Chalmers official publication statistics. To ensure that Chalmers research results are disseminated as widely as possible, an Open Access Policy has been adopted.

The CPL service is administrated and maintained by Chalmers Library. 


\section{Numerical Sensitivity of Linear Matrix Inequalities Using Shift and Delta Operators}

Bengt Lennartson Member, IEEE(*), Richard H. Middleton, Fellow, IEEE and Ivar Gustafsson

\begin{abstract}
The numerical sensitivity of Linear Matrix Inequalities (LMIs) arising from discrete-time control with short sampling periods is analyzed using shift and delta operators. The delta operator avoids cancellation problems for short sampling periods, and it includes a system scaling proportional to the inverse of the sampling period. The numerical sensitivity of both these mechanisms is investigated analytically, and verified by numerical examples. The conclusion is that the scaling procedure is (somewhat surprisingly) much more essential for shorter sampling periods than avoiding the cancellation problem.
\end{abstract}

\section{INTRODUCTION}

Linear Matrix Inequalities (LMIs) have been used extensively during the last decade. The main reason is that LMIs can be solved very efficiently by applying interior-point methods. Many signals, systems and control problems can also be formulated as convex optimization problems including LMIs, see [1], [2]. For example, various $\mathcal{H}_{\infty}$ robust control problems can be solved via a line search over a parameter, $\gamma$, of LMIs.

LMIs are formulated and solved as semidefinite programming (SDP) problems. The differential sensitivity of the optimal solution to SDP problems under perturbation of input parameters has been investigated in e.g. [3], [4]. In this paper two more specific numerical sensitivity problems are analyzed for LMIs based on discrete-time shift operator models. These sensitivity issues are related to scaling and cancellation, two well-known numerical problems for shift operator models that are conveniently solved by introducing the delta operator [5]. More recently, delta operator based LMIs have been introduced, often related to $\mathcal{H}_{\infty}$ robust control problems [6], [7].

A preliminary analysis of numerical properties of shift and delta operator LMIs was given in [8]. A more detailed analysis, including a deeper understanding of the underlying mechanisms, is given in this paper. The LMIs considered are based on the bounded real lemma, which is used to compute the induced norm from the input disturbance signal to the output performance signal. The results in this paper are easily adapted to other LMI based applications.

To obtain a natural convergence from the discrete-time to the continuous-time signal norm, both for shift and delta operator models, a signal scaling for the input and output signals is introduced. This is done in the same way as in [9], [10]. The shift operator based LMI is then shown to become nearly singular for short sampling periods, not only for the requested optimal $\gamma$-value, but also for $\gamma$-values far away from the optimal solution.

This ill-conditioned behavior generates significant errors for short sampling periods. In fact, these errors are more severe than those which arise because of the more well-known cancellation, which occurs both in LMIs and corresponding Riccati equations using shift operator models [8], [10], [5]. However, it is possible to avoid the near singularity for arbitrary $\gamma$-values by a simple scaling transformation.

B. Lennartson is with the Department of Signals and Systems, Chalmers University of Technology, Göteborg, Sweden; email: bengt.lennartson@chalmers.se (*) Three shorter sabbaticals 2003, 2006 and 2010 at the Centre for Complex Dynamic Systems \& Control, University of Newcastle, Australia are very much appreciated.

R. H. Middleton is with the School of Electrical Eng. \& Computer Science, University of Newcastle, Australia; email: Richard.Middleton@newcastle.edu.au

I. Gustafsson is with the Department of Mathematics, Chalmers University of Technology, Göteborg, Sweden; email: ivar@chalmers.se
This means that the cancellation problem can be separated, and it is then shown to be of much less importance than the singularity problem.

\section{Shift AND Delta Operator Models}

The shift and the delta operator models are briefly presented in this section, including some useful transformations between them. A natural signal scaling of the ordinary shift operator model is also introduced, related to the induced norm that will be solved in the next section.

\section{A. Shift operator model}

Consider the following discrete-time state space model on the shift operator form

$$
\left[\begin{array}{c}
q x\left(t_{k}\right) \\
y\left(t_{k}\right)
\end{array}\right]=\left[\begin{array}{cc}
A_{q} & B_{q 0} \\
C_{q 0} & D_{q}
\end{array}\right]\left[\begin{array}{l}
x\left(t_{k}\right) \\
u\left(t_{k}\right)
\end{array}\right] \triangleq G_{q 0}\left[\begin{array}{l}
x\left(t_{k}\right) \\
u\left(t_{k}\right)
\end{array}\right]
$$

where the shift operator $q$ is defined as $q x\left(t_{k}\right)=x\left(t_{k+1}\right)$. The state vector $x$, the input signal $u$ and the output signal $y$ have dimensions $n, n_{u}$ and $n_{y}$, respectively. The discrete-time updates occur at times $t_{k}, k=0,1,2, \ldots$, where the time interval between two updates is the sampling period $h=t_{k+1}-t_{k}$. The subscript 0 is included to separate this shift operator model $G_{q 0}$ from the signal scaled shift operator model $G_{q}$, which will be introduced in Section II-C and used in the rest of the paper.

\section{B. Delta operator model}

By introducing the delta operator $\delta x\left(t_{k}\right)=\left(x\left(t_{k+1}\right)-\right.$ $\left.x\left(t_{k}\right)\right) h^{-1}=\left(A_{q}-I\right) h^{-1} x\left(t_{k}\right)+B_{q 0} h^{-1} u\left(t_{k}\right)$, [5], the state space model (1) can be rewritten in delta operator form as

$$
\left[\begin{array}{c}
\delta x\left(t_{k}\right) \\
y\left(t_{k}\right)
\end{array}\right]=\left[\begin{array}{ll}
A_{\delta} & B_{\delta} \\
C_{\delta} & D_{\delta}
\end{array}\right]\left[\begin{array}{l}
x\left(t_{k}\right) \\
u\left(t_{k}\right)
\end{array}\right] \triangleq G_{\delta}\left[\begin{array}{l}
x\left(t_{k}\right) \\
u\left(t_{k}\right)
\end{array}\right]
$$

Observe that the delta operator model is an exact representation of the discrete-time system, assuming that the input signal is piecewise constant as in the shift operator model. This can be compared with e.g. the discrete Euler approximation, where the system matrix $I_{n}+h A_{c}$ is an approximation of $A_{q}$. Introducing the matrices $E=$ $\operatorname{diag}\left(I_{n}, 0\right)$ and $T_{h}=\operatorname{diag}\left(h I_{n}, I\right)$, where the dimension of the lower identity matrix in $T_{h}$ is given by the adjacent matrices, the relation between the the shift and delta operator form can be shortly expressed as $G_{q 0}=E+T_{h} G_{\delta}$.

There are two main reasons for introducing the delta operator for discrete-time models. Firstly, there is no natural transition and convergence from an ordinary discrete-time model in the shift operator $q$ to the corresponding continuous-time model. Secondly, the shift operator exhibits bad numerical behavior for short sampling periods. Both problems are naturally solved by the delta operator [5], [8].

\section{Signal scaling of the shift operator model}

Multiplying both the input signal $u$ and the output signal $y$ by a scalar does not change the input/output behavior. This is the focus when the $\mathcal{H}_{\infty}$-norm is computed in the next section by the induced norm from the input to the output signal. Observe that the system is then often a closed loop system, where the input is a disturbance signal and the output is a performance signal, while the control and measured plant outputs are internal signals. 
Compared to the model in (1), the shift operator model with the scaled input signal $u_{h}=\sqrt{h} u$ and the scaled output signal $y_{h}=$ $\sqrt{h} y$ becomes

$$
G_{q}=\left[\begin{array}{cc}
A_{q} & B_{q} \\
C_{q} & D_{q}
\end{array}\right]=\left[\begin{array}{cc}
I_{n}+h A_{\delta} & \sqrt{h} B_{\delta} \\
\sqrt{h} C_{\delta} & D_{\delta}
\end{array}\right]=E+T_{h}^{\frac{1}{2}} G_{\delta} T_{h}^{\frac{1}{2}}
$$

A consequence of this signal scaling with respect to the sampling period is that the size of the signal $y_{h}$ can be expressed by the norm $\left\|y_{h}\right\|^{2}=\sum_{k=0}^{\infty} y_{h}^{\prime}\left(t_{k}\right) y_{h}\left(t_{k}\right)=\sum_{k=0}^{\infty} y^{\prime}\left(t_{k}\right) y\left(t_{k}\right) h$ The second sum converges to a corresponding (Riemann) integral when $h \rightarrow 0$, which is in fact the main motivation for introducing the factor $\sqrt{h}$ in this state space model. Identical arguments hold for the norm of the input signal $\left\|u_{h}\right\|$.

The same type of signal scaling was used in [9], [10] for the performance output and disturbance input signals. This scaling is important to get comparable behavior when e.g. different sampling periods are evaluated. The same type of scaling also appears in traditional sampled-data control, where a continuous-time criterion is lifted to a corresponding discrete one.

\section{LineAR MATRIX INEQUALITIES}

Computation of the $\mathcal{H}_{\infty}$ norm using linear matrix inequalities (LMIs) is briefly presented in this section, both for systems on shift and delta operator form. It is well known that for a stable system $\mathcal{G}$, with input $u$ and output $y$, the $\mathcal{H}_{\infty}$ norm $\|\mathcal{G}\|_{\infty}$ is given by the induced norm $\sup _{\|u\| \neq 0} \frac{\|y\|}{\|u\|}$, cf. [11]. This norm can be calculated by solving linear matrix inequalities (LMIs) [12], [1].

\section{A. Shift operator LMI}

For the shift operator case, the following lemma shows how to solve the $\mathcal{H}_{\infty}$ norm.

Lemma 1: Consider a stable discrete-time system $\mathcal{G}$ on signal scaled shift operator form $G_{q}$ (3). The $\mathcal{H}_{\infty}$ norm $\|\mathcal{G}\|_{\infty}<\gamma$, if and only if there exists a $P=P^{\prime}>0$ such that

$$
M_{q}(P, \gamma)=\left[\begin{array}{cc}
M_{q_{11}}(P) & M_{q_{12}}(P) \\
M_{q_{12}}^{\prime}(P) & M_{q_{22}}(P, \gamma)
\end{array}\right]<0
$$

where

$$
\begin{aligned}
M_{q_{11}}(P) & =A_{q}^{\prime} P A_{q}-P+C_{q}^{\prime} C_{q} \\
M_{q_{12}}(P) & =A_{q}^{\prime} P B_{q}+C_{q}^{\prime} D_{q} \\
M_{q_{22}}(P, \gamma) & =B_{q}^{\prime} P B_{q}+D_{q}^{\prime} D_{q}-\gamma^{2} I
\end{aligned}
$$

The minimal value of $\gamma$ is obtained at the same time as the unknown $P>0$ is computed. This result is based on the bounded real lemma, see e.g. [12], where a Schur complement on a Riccati inequality gives the LMI (4).

\section{B. Delta operator LMI}

In the same way as for the shift operator case, an LMI can be formulated for delta operator models. The corresponding Riccati equation can be found in e.g. [5], [10]. Ones again, a Schur complement gives the following result.

Lemma 2: Consider a stable discrete-time system $\mathcal{G}$ in delta operator form $G_{\delta}$ (2). The $\mathcal{H}_{\infty}$ norm $\|\mathcal{G}\|_{\infty}<\gamma$, if and only if there exists a $P=P^{\prime}>0$ such that

$$
M_{\delta}(P, \gamma)=\left[\begin{array}{cc}
M_{\delta_{11}}(P) & M_{\delta_{12}}(P) \\
M_{\delta_{12}}^{\prime}(P) & M_{\delta_{22}}(P, \gamma)
\end{array}\right]<0
$$

where

$$
\begin{aligned}
M_{\delta_{11}}(P) & =A_{\delta}^{\prime} P+P A_{\delta}+h A_{\delta}^{\prime} P A_{\delta}+C_{\delta}^{\prime} C_{\delta} \\
M_{\delta_{12}}(P) & =P B_{\delta}+h A_{\delta}^{\prime} P B_{\delta}+C_{\delta}^{\prime} D_{\delta} \\
M_{\delta_{22}}(P, \gamma) & =h B_{\delta}^{\prime} P B_{\delta}+D_{\delta}^{\prime} D_{\delta}-\gamma^{2} I
\end{aligned}
$$

Similar LMI's based on the delta operator can be found in e.g. [6], [13].

Comparing the shift and delta operator matrices in (3), the block matrices (5) in $M_{q}$ are related to the corresponding delta operator matrices in (7) as $M_{q_{11}}(P)=h M_{\delta_{11}}, M_{q_{12}}(P)=\sqrt{h} M_{\delta_{12}}$, and $M_{q_{22}}(P, \gamma)=M_{\delta_{22}}$. Applying the transformation $T_{h}=$ $\operatorname{diag}\left(h I_{n}, I\right)$, we then obtain the following relation between the LMI matrices $M_{q}$ and $M_{\delta}$

$$
M_{q}(P, \gamma)=T_{h}^{\frac{1}{2}} M_{\delta}(P, \gamma) T_{h}^{\frac{1}{2}}
$$

\section{Relation between delta operator and continuous LMIs}

For a continuous-time system $G_{c}=\left[\begin{array}{cc}A_{c} & B_{c} \\ C_{c} & D_{c}\end{array}\right]$, the LMI matrix corresponding to $M_{\delta}(P, \gamma)$ is

$$
M_{c}(P, \gamma)=\left[\begin{array}{cc}
A_{c}^{\prime} P+P A_{c}+C_{c}^{\prime} C_{c} & P B_{c}+C_{c}^{\prime} D_{c} \\
B_{c}^{\prime} P+D_{c}^{\prime} C_{c} & D_{c}^{\prime} D_{c}-\gamma^{2} I
\end{array}\right]
$$

The block matrices in (7) and the fact that $G_{\delta}=G_{c}+O(h)$, cf. [8], imply that $M_{\delta}(P, \gamma)=M_{c}(P, \gamma)+O(h)$. Hence, the LMI formulation in the delta operator form converges to the corresponding continuous-time one. This is an expected but also essential convergence property in the following numerical sensitivity analysis.

\section{Numerical Sensitivity Problems}

Two numerical sensitivity problems related to the shift operator LMI $M_{q}$ will be further analyzed in this section. First, it will be shown that the LMI problem in Lemma 1 becomes ill-conditioned for short sampling periods. Secondly, a cancellation is identified in the block matrix $M_{q_{11}}(P)$ for short sampling periods.

\section{A. Ill-conditioned shift operator LMI}

LMIs are normally solved as convex optimization problems, see e.g. [2]. To show this, introduce the vector $\xi=\left[\operatorname{vec}(P)^{\prime} \gamma\right]^{\prime}$ including the unknown variables. Then consider the following semidefinite programming problem, corresponding to the LMI in Lemma 1

$$
\begin{array}{ll}
\min & \gamma \\
\text { subject to } & F(\xi)=\operatorname{diag}\left(-M_{q}(P, \gamma), P\right)>0
\end{array}
$$

This optimization problem can be solved by an interior-point method [2], where the barrier function $\phi(\xi)=-\log \operatorname{det} F(\xi)$ is introduced. The original criterion $\gamma$ is then replaced by the approximation $f(\xi)=$ $\theta \gamma+\phi(\xi)=\theta \gamma-\log \operatorname{det} F(\xi)$, where the approximation error is reduced when the parameter $\theta$ is increased. The minimization of this criterion is a convex optimization problem that can be solved by ordinary Newton type algorithms iteratively for increasing $\theta$ [2].

Starting with a feasible solution such that $F(\xi)>0$ means that $\operatorname{det} F(\xi)>0$. Decreasing $\gamma$ means that finally $\operatorname{det} F(\xi)$ gets close to zero and the barrier function $\phi(\xi)$ becomes large. At the optimum $F(\xi)$ is approximately singular and $\operatorname{det} F(\xi) \approx 0$. Hence, there is a problem when $\operatorname{det} F(\xi)$ is close to zero also when $\gamma$ is far away from the optimum, and we get an ill-conditioned optimization problem.

Since the determinant of $M_{q}$ according to (8) can be expressed as $\operatorname{det} M_{q}(P, \gamma)=\operatorname{det}\left(T_{h}^{\frac{1}{2}} M_{\delta}(P, \gamma) T_{h}^{\frac{1}{2}}\right)=\operatorname{det} T_{h} \operatorname{det} M_{\delta}(P, \gamma)=$ $h^{n} \operatorname{det} M_{\delta}(P, \gamma)$, we find that this ill-conditioned behavior occurs for short sampling periods, since

$$
\begin{aligned}
\operatorname{det} F(\xi) & =\operatorname{det} \operatorname{diag}\left(-M_{q}(P, \gamma), P\right) \\
& =(-1)^{n+n_{u}} h^{n} \operatorname{det} M_{\delta}(P, \gamma) \operatorname{det} P
\end{aligned}
$$


is close to zero independently of $\gamma$ when the sampling period $h$ is small.

Hence, the shift operator LMI becomes ill-conditioned for short sampling periods. Since the singularity of $M_{q}$ is expected to be a unique property of the optimal solution, it is hard to find the exact minimal value of $\gamma$ when this property is not fulfilled. This will also be confirmed in the numerical investigation in Section VI, where a number of SDP solvers are unable to solve the shift operator LMI $M_{q}<0$ for short sampling periods.

The relation (8) suggests however to scale $M_{q}$ with $T_{h}^{-\frac{1}{2}}$ on both sides, to avoid the singularity problem for $M_{q}$. We therefore introduce the following scaled LMI problem

$$
M_{S}(P, \gamma)=T_{h}^{-\frac{1}{2}} M_{q}(P, \gamma) T_{h}^{-\frac{1}{2}}<0
$$

which gives the same optimal $\gamma$ as the shift operator LMI, but without the singularity problem for small sampling periods.

As long as the corresponding continuous-time system $G_{c}$ is well behaved, the continuous-time LMI solution can be computed without numerical problems. Because $M_{\delta}(P, \gamma) \rightarrow M_{c}(P, \gamma)$ when $h \rightarrow$ 0 , the same can be expected for the delta operator LMI solution, especially for shorter sampling periods. Since algebraically $M_{S}=$ $M_{\delta}$, this means that the scaled LMI problem also can be expected to behave well for shorter sampling periods. However, one problem remains and that is the cancellation in both $M_{q}$ and $M_{S}$. This will be discussed in the next subsection.

\section{B. Cancellation in shift operator LMI}

The block matrix $M_{q_{11}}=A_{q}^{\prime} P A_{q}-P+C_{q}^{\prime} C_{q}$ in (5) includes a cancellation for short sampling periods, since then $A_{q}=I+O(h)$ and $M_{q 11}=(I+O(h))^{\prime} P(I+O(h))+h C_{\delta}^{\prime} C_{\delta}-P=P_{h}-P+$ $O(h)=O(h)$, where $P_{h} \approx P$. This cancellation can be avoided either by using the delta operator matrix $M_{\delta}$, or simply be replacing the block matrix $M_{q_{11}}$ in $M_{q}$ by $M_{\Delta_{11}}=A_{\Delta}^{\prime} P+P A_{\Delta}+A_{\Delta}^{\prime} P A_{\Delta}+$ $C_{q}^{\prime} C_{q}$ where $A_{\Delta}=h A_{\delta}\left(A_{q}=I+A_{\Delta}\right)$. The LMI $M_{q}(P, \gamma)<0$ is then replaced by

$$
M_{\Delta}(P, \gamma)=\left[\begin{array}{cc}
M_{\Delta_{11}}(P) & M_{q_{12}}(P) \\
M_{q_{12}}^{\prime}(P) & M_{q_{22}}(P, \gamma)
\end{array}\right]<0
$$

Note that algebraically $M_{\Delta}=M_{q}$, but numerically the cancellation problem is avoided in $M_{\Delta}$. In fact, we have now separated the two properties of the delta operator from an LMI perspective. The cancellation is avoided in $M_{\Delta}(12)$, and the system scaling, including the $1 / h$ factor in both $A_{\delta}$ and in the delta operator, is introduced in $M_{S}$. In the following two sections an error analysis for all four LMI problems $M_{q}<0, M_{\Delta}<0, M_{S}<0$, and $M_{\delta}<0$ will be given, both analytically and numerically.

\section{ERROR ANALYSIS}

We will now investigate the numerical error in the computation of the LMI matrices $M_{q}, M_{\Delta}, M_{S}$, and $M_{\delta}$. In particular, the contribution from the cancellation in $M_{q}$ and $M_{S}$ will be analyzed as a function of the sampling period $h$.

\section{A. Errors in the LMI matrices}

Motivated by floating point arithmetic implementations, we will use a relative error analysis [14]. It is well known that subtraction between two uncertain numbers being almost equal yields cancellation of digits. To be more precise, let $a^{\epsilon}$ and $b^{\epsilon}$ be stored representations of two numbers $a$ and $b$. Then $a^{\epsilon}=\left(1+\epsilon_{a}\right) a$ and $b^{\epsilon}=\left(1+\epsilon_{b}\right) b$, where $\left|\epsilon_{a}\right| \leq \mu$ and $\left|\epsilon_{b}\right| \leq \mu$, and $\mu$ is the machine precision $\left(\mu \approx 2 \cdot 10^{-16}\right.$ in MATLAB $)$. Now assume that the subtraction is performed according to the IEEE-standard, [14]. Then the stored result is $(a-b)^{\epsilon}=\left(1+\epsilon_{s}\right)\left(a^{\epsilon}-b^{\epsilon}\right)$ with $\left|\epsilon_{s}\right| \leq \mu$.

For matrices similar expressions can be formulated introducing the Hadamard (entry-wise) matrix multiplication [15], the one matrix $\mathbf{1}$, and the relative error matrix $\varepsilon$, where $[\mathbf{1}]_{i j}=1$ and $[\varepsilon]_{i j}=\epsilon_{i j}$. Then $A^{\epsilon}=\left(\mathbf{1}+\varepsilon_{A}\right) \circ A$ and $(A-B)^{\epsilon}=\left(\mathbf{1}+\varepsilon_{s}\right) \circ\left(\left(\mathbf{1}+\varepsilon_{A}\right) \circ\right.$ $\left.A-\left(\mathbf{1}+\varepsilon_{B}\right) \circ B\right)$. Neglecting the quadratic error terms $\varepsilon_{s} \circ \varepsilon_{A} \circ A$ and $\varepsilon_{s} \circ \varepsilon_{B} \circ B$, the error in the matric subtraction can be expressed as

$$
(A-B)^{\epsilon}-(A-B)=\left(\varepsilon_{s}+\varepsilon_{A}\right) \circ(A-B)+\left(\varepsilon_{A}-\varepsilon_{B}\right) \circ B
$$

where the asymmetry between $A$ and $B$ is introduced to fit the analysis of the cancellation in $M_{q_{11}}$. Introducing the corresponding entry-wise division, using the notation $\phi$, the relative error becomes

$\left((A-B)^{\epsilon}-(A-B)\right) \phi(A-B)=\left(\varepsilon_{s}+\varepsilon_{A}\right)+\left(\varepsilon_{A}-\varepsilon_{B}\right) \circ B \phi(A-B)$

Obviously this error becomes large when the elements in $A-B$ are small. Remind that the elements in the error matrices $\varepsilon_{*}$ are no larger in magnitude than $\mu$. Based on this result we consider two cases:

1) The matrix $A-B$ becomes small when the sampling period $h \rightarrow 0$, i.e. $A-B=O(h)$, while $A=O(1)$ and $B=O(1)$. According to (14) the elements of $(A-B)^{\epsilon}$ then have a relative error of size $O\left(\mu+\mu h^{-1}\right)$ for small $h$.

2) The matrix $A-B=O(1)$ for all sampling periods. Then the elements of $(A-B)^{\epsilon}$ have a relative error of size $O(\mu)$ independently of $h$.

This error analysis is now applied to the LMI matrices $M_{q}, M_{\delta}$, $M_{S}$, and $M_{\Delta}$. For $M_{q_{11}}$ in (5), let $A=A_{q}^{\prime} P A_{q}+C_{q}^{\prime} C_{q}$ and $B=P$, which leads to $A-B=M_{q_{11}}$. Since the analysis is focused on the cancellation, it is assumed for simplicity that $A=A_{q}^{\prime} P A_{q}+C_{q}^{\prime} C_{q}$ is computed without error, but stored with a relative error as well as $P$. Based on (13), the error in the computation of $M_{q_{11}}$ can then be expressed as

$$
M_{q 11}^{\epsilon}(P)=M_{q 11}(P)+\varepsilon_{q_{11}} \circ M_{q 11}(P)+\varepsilon_{P} \circ P
$$

where $\varepsilon_{q_{11}}=O(\mu)$ and $\varepsilon_{P}=O(\mu)$. Since $M_{q_{11}}=A-B=O(h)$ according Section IV-B, while $P=O(1)$, the elements of $M_{q_{11}}^{\epsilon}$ have a relative error, according to case one above, of size $O\left(\mu+\mu h^{-1}\right)$ for small $h$. This highlights the effect of the cancellation when $h \rightarrow 0$.

The other block matrices in $M_{q}$ are computed according to case two above without any cancellation, and can therefore be simplified to $M_{q_{12}}^{\epsilon}(P)=M_{q_{12}}(P)+\varepsilon_{q_{12}} \circ M_{q_{12}}(P)$ and $M_{q_{22}}^{\epsilon}(P, \gamma)=$ $M_{q_{22}}(P, \gamma)+\varepsilon_{q_{22}} \circ M_{q_{22}}(P, \gamma)$, where the relative errors $\varepsilon_{q_{12}}$ and $\varepsilon_{q_{22}}$ are of size $O(\mu)$. Introduce the error matrix due to the cancellation in $M_{q_{11}}$

$$
P^{\epsilon}=\operatorname{diag}\left(\varepsilon_{P} \circ P, 0_{n_{u} \times n_{u}}\right)
$$

Since $P=O(1)$, we observe that $P^{\epsilon}=O(\mu)$. Together with (15), $M_{q}^{\epsilon}(P, \gamma)$ can now be formulated as $M_{q}^{\epsilon}(P, \gamma)=M_{q}(P, \gamma)+\varepsilon$ 。 $M_{q}(P, \gamma)+P^{\epsilon}$, where $\varepsilon=\left[\begin{array}{ll}\varepsilon_{q_{11}} & \varepsilon_{q_{12}} \\ \varepsilon_{q_{12}}^{\prime} & \varepsilon_{q_{22}}\end{array}\right]=O(\mu)$. Applying the transformation (8) finally gives

$$
M_{q}^{\epsilon}(P, \gamma)=T_{h}^{\frac{1}{2}}\left(M_{\delta}(P, \gamma)+\varepsilon \circ M_{\delta}(P, \gamma)+P^{\epsilon} / h\right) T_{h}^{\frac{1}{2}}
$$

Similar analysis shows that the errors in $M_{\delta}$ and $M_{\Delta}$ can be expressed in the same way, but without the last term $P^{\epsilon}$, since there is no cancellation involved in these LMIs. For $M_{S}$ the error term $P^{\epsilon}$ due to the cancellation remains, while the transformation $T_{h}^{\frac{1}{2}}$ disappears as well as in $M_{\delta}$. By these remarks we are ready to formulate the following theorem. 
Theorem 3: Assume that $M_{\delta}$ is computed and stored with relative error $\varepsilon$ as

$$
M_{\delta}^{\epsilon}(P, \gamma)=M_{\delta}(P, \gamma)+\varepsilon \circ M_{\delta}(P, \gamma)
$$

where the relative error matrix $\varepsilon=O(\mu)$. The corresponding errors in $M_{q}, M_{\Delta}$ and $M_{S}$ can then be expressed as

$$
\begin{aligned}
M_{q}^{\epsilon}(P, \gamma) & =T_{h}^{\frac{1}{2}}\left(M_{\delta}^{\epsilon}(P, \gamma)+P^{\epsilon} / h\right) T_{h}^{\frac{1}{2}} \\
M_{\Delta}^{\epsilon}(P, \gamma) & =T_{h}^{\frac{1}{2}} M_{\delta}^{\epsilon}(P, \gamma) T_{h}^{\frac{1}{2}} \\
M_{S}^{\epsilon}(P, \gamma) & =M_{\delta}^{\epsilon}(P, \gamma)+P^{\epsilon} / h
\end{aligned}
$$

where the error matrix due to the cancellation $P^{\epsilon}=O(\mu)$.

This theorem highlights the fact that there are mainly two error sources in the shift operator based LMI calculations. The first has to do with the bad scaling for shorter sampling periods, expressed by the transformation matrix $T_{h}^{\frac{1}{2}}$ and present in $M_{q}$ as well as in $M_{\Delta}$. Due to $T_{h}^{\frac{1}{2}}$ in (18) and (19) the LMI problem becomes illconditioned, where the LMI matrices $M_{q}$ and $M_{\Delta}$ becomes near singular independently of $\gamma$ when $h \rightarrow 0$, according to the analysis in Section IV-A. The other error source is the cancellation in $M_{q}$ and $M_{S}$, resulting in the error term $P^{\epsilon} / h$. Both these error sources are avoided in the delta operator form $M_{\delta}$.

\section{B. Error sensitivity in the objective function}

To investigate in more detail how the cancellation error influences the optimization, first assume that the objective function in (10) depends on an error vector $\epsilon$ included in the constraint function $F(\xi, \epsilon)>0$, that is $f(\xi, \epsilon)=\theta \gamma-\log \operatorname{det} F(\xi, \epsilon)$ Then the partial derivative

$$
\begin{aligned}
\frac{\partial f(\xi, \epsilon)}{\partial \epsilon_{i}} & =-\frac{1}{\operatorname{det} F(\xi, \epsilon)} \frac{\partial}{\partial \epsilon_{i}} \operatorname{det} F(\xi, \epsilon) \\
& =-\operatorname{tr}\left(F^{-1}(\xi, \epsilon) \frac{\partial}{\partial \epsilon_{i}} F(\xi, \epsilon)\right)
\end{aligned}
$$

This partial derivative is now analyzed for the scaled LMI problem $M_{S}<0$, which only includes the cancellation error but not the near singularity problem. The error term due to the cancellation $P^{\epsilon} / h$, defined in (16), depends on the error matrix $\varepsilon_{P}$. Hence, we investigate (21) with respect to the elements in this matrix $\epsilon_{p_{i j}}=\left[\varepsilon_{P}\right]_{i, j}$. First consider (20), where we only include the error matrix $\varepsilon_{P}$ but not $\varepsilon$ in $M_{\delta}^{\epsilon}$. This means that $M_{\delta}^{\epsilon}$ in (20) is simplified to $M_{\delta}=M_{S}$, and we obtain

$$
\begin{aligned}
F\left(\xi, \varepsilon_{P}\right) & =\operatorname{diag}\left(-M_{S}(P, \gamma)-P^{\epsilon} / h, P\right) \\
& =\operatorname{diag}\left(-M_{S}(P, \gamma)-(1 / h) \operatorname{diag}\left(\varepsilon_{P} \circ P, 0_{n_{u} \times n_{u}}\right), P\right)
\end{aligned}
$$

which gives

$$
\left[\frac{\partial F\left(\xi, \varepsilon_{P}\right)}{\partial \epsilon_{p_{i j}}}\right]_{k, l}= \begin{cases}-\frac{p_{i j}}{h} & k=i, \ell=j \\ 0 & \text { otherwise }\end{cases}
$$

where $p_{i j}=[P]_{i j}$. Since the inverse $F\left(\xi, \varepsilon_{P}\right)^{-1}=\operatorname{diag}($ $\left.-\left(M_{S}(P, \gamma)+P^{\epsilon} / h\right)^{-1}, P^{-1}\right)$, the partial derivative of the objective function (21) can now be expressed as

$$
\frac{\partial f\left(\xi, \varepsilon_{P}\right)}{\partial \epsilon_{p_{i j}}}=-\left[\left(M_{S}(P, \gamma)+\frac{P^{\epsilon}}{h}\right)^{-1}\right]_{j, i} \frac{p_{i j}}{h}
$$

This result is used in the following theorem.

Theorem 4: For the scaled LMI problem $M_{S}<0$ the sensitivity of the approximative objective function $f(\xi)=\theta \gamma-\log \operatorname{det} F(\xi)$, with respect to the cancellation errors $\epsilon_{p_{i j}}=\left[\varepsilon_{P}\right]_{i, j}$ in $P^{\epsilon}$, can approximately be determined as

$$
f\left(\xi, \epsilon_{p_{i j}}\right) \approx f(\xi, 0)+\left.\epsilon_{p_{i j}} \frac{\partial f\left(\xi, \varepsilon_{P}\right)}{\partial \epsilon_{p_{i j}}}\right|_{\varepsilon_{P}=0}=f(\xi, 0)+\epsilon_{p_{i j}} \frac{c_{i j}}{h}
$$

for $i, j=1, \ldots, n$, where $c_{i j}=-p_{i j}\left[M_{S}(P, \gamma)^{-1}\right]_{j, i}$

Observe that $f(\xi, 0)$ becomes large at the optimum due to a large $\theta$, but also $c_{i j}$, since it includes the inverse of $M_{S}$ that is near-singular at the optimum. This is valid for arbitrary sampling periods, which shows that the objective function will be sensitive to the error in $P^{\epsilon}$ for sufficiently small sampling periods.

Now introduce a relative error in the determination of the optimal $\gamma$ value for the actual LMI problems

$$
e_{\gamma}=\frac{\left|\gamma-\gamma_{0}\right|}{\gamma_{0}}
$$

where $\gamma_{0}$ is the true optimal value and $\gamma$ is the optimal value computed by the different LMIs. As already observed, for the scaled LMI problem $M_{S}<0$, the objective function becomes more and more sensitive to the error in $P^{\epsilon}$ due to cancellation for shorter sampling periods, according to Theorem 4 . Since this error sensitivity is proportional to $1 / h$, and the determination of the optimal $\gamma$ value is based on this objective function, the relative error $e_{\gamma}^{S}$ for the LMI $M_{S}<0$ is also expected to increase with the same factor $1 / h$, that is

$$
e_{\gamma}^{S} \approx \frac{\epsilon_{S}}{h} \text { for small } h
$$

where $\epsilon_{S}$ is a constant factor. This error function is verified in the next section, where four different SDP solvers generate the same error behavior for shorter sampling periods $h$, and the value of $\epsilon_{S}$ is shown to be of the same order as the machine precision.

\section{NUMERICAL ILLUSTRATIONS}

Consider the following resonant dynamic system with continuoustime transfer function

$$
G_{c}(s)=\frac{12}{(s+1)\left(s^{2}+0.2 s+1\right)\left(s^{2}+0.4 s+4\right)}
$$

where the accuracy in the computation of the corresponding discretetime $\mathcal{H}_{\infty}$ norm will be evaluated. This is done by solving the four different LMIs considered in this paper for various sampling periods $h \leq 1$ using different SDP solvers.

First a balanced state-space model is generated using the MATLAB function ssbal. Corresponding discrete-time state-space models with zero-order hold circuit at the control input are then computed for different sampling periods, both in shift and delta operator versions. Finally, the minimal $\gamma$ value is computed for the different LMIs $M_{q}<0$ (4), $M_{\delta}<0$ (8), $M_{\Delta}<0$ (12) and $M_{S}<0$ (11), resulting in corresponding relative errors $e_{\gamma}^{q}, e_{\gamma}^{\delta}, e_{\gamma}^{\Delta}$, and $e_{\gamma}^{S}$.

The LMIs are solved by four different semi definite programming (SDP) solvers SeDuMi [16], SDPT3[17], SDPA [18], and PENSDP [19]. They are all run on top of MATLAB via the user friendly interface YALMIP [20].

In order to obtain a correct relative error $e_{\gamma}$ (22) the true gamma value $\gamma_{0}$ is determined by the maximum frequency response based on the delta operator with a very dense grid (20000 points around the maximum at $\approx 1 \mathrm{rad} / \mathrm{s}$ ). As an extra check, this result is compared to the continuous-time infinity norm function in MATLAB for very short sampling periods, and the corresponding discrete one for longer sampling periods, with a very small relative difference around $10^{-11}$.

The resulting relative errors $e_{\gamma}^{S}$ for the scaled LMI $M_{S}<0$ are shown in Fig. 1 for the different solvers. Since the cancellation remains in $M_{S}$ the error $e_{\gamma}^{S}$ increases for shorter sampling periods for all four solvers approximately as $\epsilon_{S} / h$, the thin line in Fig. 1. The constant $\epsilon_{S} \approx 2 \cdot 10^{-17}$, which is of the same order as the machine precision in MATLAB $\left(2 \cdot 10^{-16}\right)$. This is reasonable since the error source is a cancellation. The result also coincide with the analysis in Section IV and especially Theorem 4 and (23). 


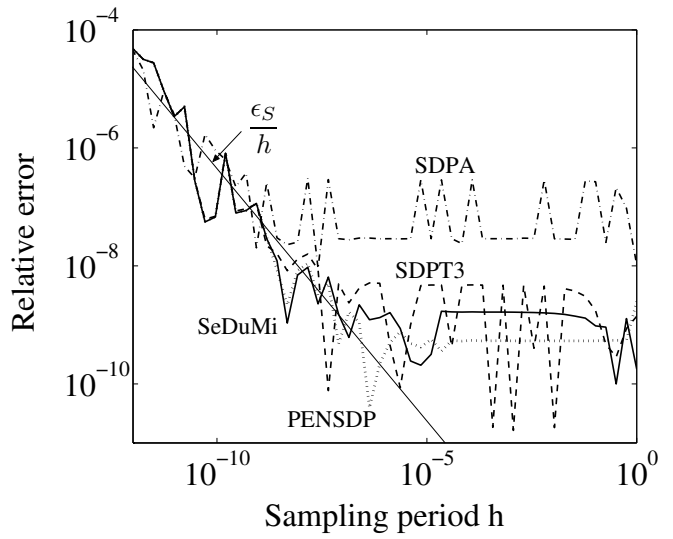

Fig. 1 Relative error for the SDP solvers SeDuMi (solid), SDPT3 (dashed), PENSDP (dotted), and SDPA (dashdot), when $\gamma$ is minimized for the scaled LMI $M_{S}<0$.

Furthermore, note that for longer sampling periods the relative error depends more on the general accuracy of the individual LMI solver. No adjustments of the tuning parameters for the different solvers have been performed, and we observe that the solver SDPA is generally tuned to achieve less relative accuracy $\left(\approx 10^{-7}\right)$ than the other solvers.

Corresponding relative errors for the delta operator case agree with the more constant behavior of $e_{\gamma}^{S}$ for longer sampling periods, with $e_{\gamma}^{\delta}$ around $10^{-7}$ for SDPA and $10^{-8}-10^{-9}$ for the rest of the solvers, with a few outliers up to $10^{-7}$ for SDPT3. The difference is that the delta operator LMI behaves equally well also for very short sampling periods. For all practical choices of sampling periods we find on the other hand that the scaling mechanism in $M_{S}$ is sufficient to achieve perfect numerical results. The cancellation error simply shows up only for unrealistically short sampling periods.

The ill-conditioned behavior in both $M_{q}$ and $M_{\Delta}$ is however shown to be much more severe. The relative errors for the shift operator case $e_{\gamma}^{q}$ are shown in Fig. 2. It is evident that the solvers have great difficulties to generate accurate results, in the case of SDPA already at $h=0.0035$ and for PENSDP at $h=0.001$. The most robust solver here seems to be SDPT3, which generates accurate

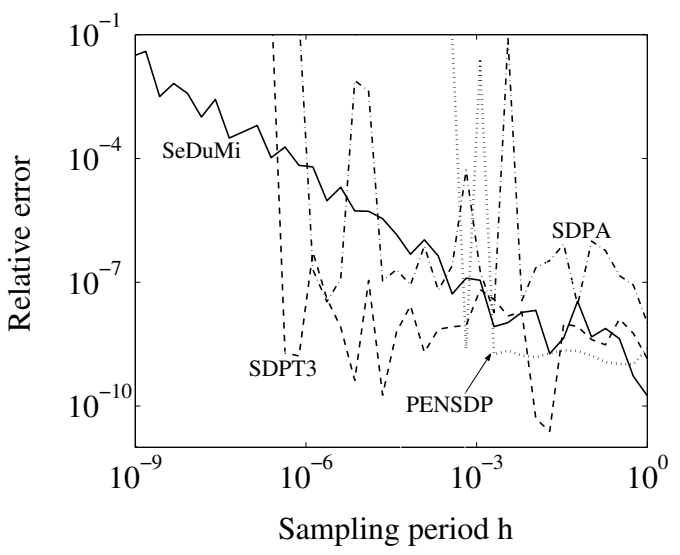

Fig. 2 Relative error for the SDP solvers SeDuMi (solid), SDPT3 (dashed), PENSDP (dotted), and SDPA (dashdot), when $\gamma$ is minimized for the shift operator LMI $M_{q}<0$. result down to $h=10^{-6}$. For the SeDuMi solver the relative error $e_{\gamma}^{q}$ increases as $1 / h$ in the same way as the cancellation error. To summarize the singularity problem results in much more diversified error behaviors, more dependent on algorithmic details in the different solvers.

The removal of the cancellation in $M_{\Delta}$ does not change its error behavior. The same severe errors as for the shift operator LMI are shown for shorter sampling periods. The reason is that the cancellation error according to Fig. 1 appears at much shorter sampling periods than the error caused by the singularity problem, still included in $M_{\Delta}$.

We also observe that $M_{\Delta}^{\epsilon}=T_{h}^{\frac{1}{2}} M_{\delta}^{\epsilon}(P, \gamma) T_{h}^{\frac{1}{2}}$ and $M_{q}^{\epsilon}=$ $T_{h}^{\frac{1}{2}} M_{S}^{\epsilon}(P, \gamma) T_{h}^{\frac{1}{2}}$ according to Theorem 3. Since both the delta operator and the scaled LMIs work well also for shorter sampling periods (except very small $h$ for the scaled LMI), it is obvious that the bad error behavior for shorter sampling periods related to both $M_{q}$ and $M_{\Delta}$ is purely caused by the singularity problem represented by $T_{h}^{\frac{1}{2}}$.

To conclude, a numerically robust solution for shorter sampling periods is to use the delta operator model, or to introduce the simple scaling mechanism in $M_{S}=T_{h}^{-\frac{1}{2}} M_{q} T_{h}^{-\frac{1}{2}}$. Both approaches work fine for all practical choices of sampling periods.

\section{CONCLUSIONS}

Numerical properties have been analyzed when the $\mathcal{H}_{\infty}$ norm is calculated for discrete-time systems by Linear Matrix Inequalities (LMIs). In particular, the behavior for shorter sampling periods has been investigated. By analysis and numerical illustrations it has been shown that there are two main error sources when systems are modeled by the ordinary discrete-time shift operator. The quite well-known cancellation problem in the shift operator case is shown to be less important compared to the fact the LMI problem is fundamentally ill-conditioned for shorter sampling periods $h$. In a study of a numerical example, four different numerical solvers for LMIs exhibit problems with this ill-conditioned behavior, two of them with severe errors for sampling periods that may appear in real applications.

All these numerical problems are solved by using a delta operator formulation of the LMI. Alternatively, it is possible to use the shift operator model but then apply a simple scaling transformation on the LMI. This transformation captures the system scaling mechanism in the delta operator model, but does not avoid the cancellation problem. The relative error then however becomes negligible for all practical choices of sampling periods.

\section{REFERENCES}

[1] S. Boyd, L. E. Ghaoui, E. Feron, and V. Balakrishnan, Linear Matrix Inequalities in System and Control Theory. SIAM Studies in Applied Mathematics, 1994.

[2] S. Boyd and L. Vandenberghe, Convex Optimization. Cambridge University Press, 2004.

[3] S. A. Miller, "Sensitivity of solutions to semidefinite programs, Tech. Rep. CCEC-97-0519, http://www-ccec.ece.ucsb.edu/techrpts/report-970519.ps, 1997.

[4] J. F. Sturm and S. Zhang, "On sensitivity of central solutions in semidefinite programming," Mathematical Programming, vol. 90, no. 2, pp. 205-227, 2001.

[5] R. H. Middleton and G. C. Goodwin, Digital Control and Estimation A Unified Approach. Prentice Hall, Englewood Cliffs, N. J., 1990.

[6] E. Collins, W. Haddad, V. Chellaboina, and T. Song, "Robustness analysis in the delta-domain using fixed-structure multipliers," in Proc. 36th IEEE Conference on Decision and Control, San Diego, December 1997, pp. 3286-91. 
[7] R. Lin, F. Yang, and Q. Chen, "Design of robust non-fragile $\mathcal{H}_{\infty}$ controller based on delta operator theory," Journal of Control Theory and Applications, vol. 5, no. 4, pp. 404-408, 2007.

[8] B. Lennartson, R. H. Middleton, A. K. Christiansson, and T. McKelvey, "Low order sampled data $\mathcal{H}_{\infty}$ control using the delta operator and LMIs," in Proc. 43rd IEEE Conference on Decision and Control, Bahamas, December 2004, pp. 4479-4484.

[9] M. Salgado, R. H. Middleton, and G. C. Goodwin, "Connection between continuous and discrete Riccati equations with applications to Kalman filtering," IEE Proceedings, Part D, vol. 135, no. 1, pp. 28-34, 1988.

[10] B. Lennartson, R. H. Middleton, and A. K. Christiansson, "Unified, periodic \& sampled data $\mathcal{H}_{\infty}$ control using the delta operator," in Proc. 43rd IEEE Conference on Decision and Control, Bahamas, December 2004, pp. 2364-2369.

[11] M. Green and D. Limebeer, Linear Robust Control. Prentice Hall, Englewood Cliffs, N. J., 1995.

[12] P. Gahinet and P. Apkarian, "A linear matrix inequality approach to $\mathcal{H}_{\infty}$ control," International Journal on Robust and Nonlinear Control, vol. 4, pp. 421-448, 1994.

[13] H. Lee, J. Park, and Y. Joo, "Further refinement on LMI-based digital redesign: delta-operator approach," IEEE Transactions on Circuits and Systems Part II: Express Briefs, vol. 53, no. 6, pp. 473-477, 2006.

[14] B. N. Datta, Numerical Linear Algebra and Applications, 2nd ed. SIAM, 2010.

[15] R. A. Horn and C. R. Johnson, Topics in matrix analysis. Cambridge University Press, 1994.

[16] J. F. Sturm, "Using SeDuMi 1.02, a MATLAB toolbox for optimization over symmetric cones," Optimization Methods and Software, 1999. SeDuMi URL: http://sedumi.ie.lehigh.edu.

[17] R. H. Tutuncu, K. C. Toh, and M. J. Todd, "Solving semidefinitequadratic-linear programs using SDPT3," Mathematical Programming Series $B$, vol. 95, pp. 189-217, 2003.

[18] M. Kojima, K. Fujisawa, K. Nakata, and M. Yamashita, "SDPA (SemiDefinite Programming Algorithm) User's Manual - Version 6.20.” Dept. of Mathematical and Computing Sciences, Tokyo Institute of Technology, Tech. Rep., 2005. SDPA URL: http://sdpa.indsys.chuo-u.ac.jp/sdpa.

[19] M. Kocvara and M. Stingl, "On the solution of large-scale SDP problems by the modified barrier method using iterative solvers," Mathematical Programming Series B, vol. 109, no. 2-3, pp. 413-444, 2007. PENSDP URL: http://www.penopt.com.

[20] J. Löfberg, "YALMIP: a toolbox for modeling and optimization in MATLAB," in Proc. 2004 IEEE International Symposium on Computer Aided Control Systems Design, Taipei, September 2004, p. 284. YALMIP URL: http://users.isy.liu.se/johanl/yalmip/. 